\title{
Mosquito traps designed to capture Aedes aegypti (Diptera: Culicidae) females: preliminary comparison of Adultrap, MosquiTRAP and backpack aspirator efficiency in a dengue-endemic area of Brazil
}

\author{
Rafael Maciel-de-Freitas/ ${ }^{+}$, Roberto Costa Peres, Fernando Alves ${ }^{1}$, Mauro Blanco Brandolini ${ }^{1}$ \\ Laboratório de Transmissores de Hematozoários, Instituto Oswaldo Cruz-Fiocruz, Av. Brasil 4365, 21045-900 Rio de Janeiro, RJ, Brasil \\ ${ }^{1}$ Secretaria Municipal de Saúde do Rio de Janeiro, Rio de Janeiro, RJ, Brasil
}

In this report, the efficiency of Adultrap under field conditions is compared to a CDC backpack aspirator and to MosquiTRAP. An urban dengue-endemic area of Rio de Janeiro was selected to evaluate the efficiency of mosquito traps in capturing Aedes aegypti females. Adultrap and aspirator captured similar numbers of Ae. aegypti females, with the former showing high specificity to gravid individuals (93.6\%). A subsequent mark-release-recapture experiment was conducted to evaluate Adultrap and MosquiTRAP efficiency concomitantly. With a $6.34 \%$ recapture rate, MosquiTRAP captured a higher mean number of female Ae. aegypti per trap than Adultrap $\left(\chi^{2}=14.26\right.$; $d f=1$; $p<0,05)$. However, some MosquiTRAPs (28.12\%) contained immature Ae. aegypti after 18 days of exposure in the field and could be pointed as an oviposition site for female mosquitoes. Both trapping methods, designed to collect gravid Ae. aegypti females, seem to be efficient, reliable and may aid routine Ae. aegypti surveillance.

Key words: dengue - oviposition trap - vector surveillance - container productivity

In Brazil, field monitoring of Aedes aegypti populations is currently focused on the inspection of sampled houses for immature stages 4-6 times per year (Braga et al. 2003). However, this method is labor-intensive, not very sensitive and subject to both the efficiency and motivation of the health agent and obtaining the permission of the homeowner. Taken together, these drawbacks can result in imprecise and low-confidence estimations of infestation indices (Focks 2003).

At present, dengue transmission can only be reduced or interrupted by controlling its mosquito vector, Ae. aegypti. Several methods for sampling the adult mosquito population, such as mosquito traps, have been recently developed worldwide (Silver 2008). Different methods vary in efficiency and labor required: the BG-Sentinel is more efficient in collecting Ae. aegypti females than a backpack aspirator, but both methods may be too laborious to permit daily mosquito collection in dengueendemic areas (Maciel-de-Freitas et al. 2006, Williams et al. 2006). In Brazil, major efforts have been directed to develop a mosquito trap that will specifically collect gravid Ae. aegypti females. During the last decade, two such traps have been produced: Adultrap and MosquiTRAP. Until now, the efficiencies of Adultrap and MosquiTRAP have been compared with aspiration and other methods, but never with one another in the same space and time (Gomes et al. 2007, Gama et al. 2007). In addition, little information on Adultrap is available in the literature, probably due to its recent development.

+ Corresponding author: freitas@ioc.fiocruz.br

Received 9 April 2008

Accepted 26 August 2008
Thus, the main objective of this report is to evaluate the efficiency of the Adultrap in capturing Ae. aegypti females, comparing it to both a backpack aspirator and MosquiTRAP in field trials in an urban neighborhood of Rio de Janeiro.

The field work was performed in a suburban neighborhood of Rio de Janeiro, named Olaria (22 $50^{\prime} 29^{\prime \prime} \mathrm{S}$ $43^{\circ} 15^{\prime} 59^{\prime \prime} \mathrm{W}$ ), which has regular water supply and garbage collection, low vegetation cover and well-defined and planned blocks dominated by residential buildings (3-4 dorm rooms). Olaria showed a House Index (HI) (number of positive houses/ number of inspected houses) of 8.8 one month prior to the beginning of experiments. In 2007, 212 dengue cases were confirmed in Olaria; 467 were confirmed from January through the first week of July 2008 (SMS 2008).

Adultrap was designed to capture gravid Ae. aegypti females during oviposition, using water as its principal attractant. Water remains confined in a compartment at the bottom of the trap that cannot be reached by trapped mosquitoes, avoiding egg laying. A large hole on the top is the principal entrance of attracted females, which become trapped in an interior chamber (Gomes et al. 2007). In the first stage of evaluations, we compared Adultrap's efficiency and sensitivity to that of a backpack aspirator, the gold standard collection method, and tested whether Adultraps baited with hay infusion had a higher collection efficiency than those baited with water alone.

In field trials, 100 houses were randomly selected to have an Adultrap installed, 50 traps with only water as an attractant and another 50 traps with water plus hay infusion. Trap characteristics such as location, luminosity and bait type were recorded daily. One hundred houses were selected for aspiration using a CDC backpack aspirator (15-20 min per house, in intra- and peridomestic environments), following Maciel-de-Freitas et al. (2006). 
Fifty of the selected houses had an Adultrap installed and 50 had no Adultrap. Of the 50 houses selected for aspiration that had an Adultrap installed, 25 had only water and 25 had water plus hay infusion. Trap monitoring and aspiration was performed daily for 11 days, starting on August 22nd 2006. There were no aspiration and trap monitoring on days 5, 6 and 7 due to technical and personnel limitations; aspiration was not performed on day 8 due to intense rain.

The ovaries of collected Ae. aegypti females were dissected to evaluate Adultrap's sensitivity in capturing females in a specific physiological state. Ovarian development was classified according to Christophers (1911). Ovaries in stages I, I-II and II were grouped as initial development, stages III and IV grouped as intermediary stages and stage $\mathrm{V}$ classified as final stage, when females would be considered gravid. To evaluate if Adultrap collects all gravid females in a single house, we dissected the ovaries of Ae. aegypti captured by aspiration in houses with an Adultrap installed. The ovaries of Ae. aegypti collected in houses without Adultrap were also dissected to determine if results were the consequence of a naturally higher proportion of gravid females in Olaria during field experiments.

Two mosquito species were collected: Ae. aegypti and Culex quinquefasciatus (Table, data for Ae. Aegypti only). Statistically, Adultrap and aspirator collect similar mean numbers of Ae. aegyptifemales $(\mathrm{t}=0.93 ; \mathrm{df}=13 ; \mathrm{p}=0.372)$, but the former is more efficient in capturing gravid individuals $(\mathrm{t}=4.26 ; \mathrm{df}=13 ; \mathrm{p}<0.05)$. No significant difference was observed between the mean numbers of Ae. aegypti females collected in Adultraps set in the peridomestic area and inside houses $(\mathrm{t}=0.106 ; \mathrm{df}=54 ; \mathrm{p}=$ $0.915)$; set undersunlight or in the shadow $(\mathrm{t}=1.39$; $\mathrm{df}=54$; $\mathrm{p}=0.17)$, or when baited with water or with both water and hay infusion $(\mathrm{t}=1.69 ; \mathrm{df}=54 ; \mathrm{p}=0.095)$.

\section{TABLE}

Total and mean number of Aedes aegypti captured daily in 100 houses with an Adultrap and in 100 houses with aspirator collections in the neighborhood of Olaria, August 22nd-September 1st 2006. There were no collections on days 5, 6 and 7 due to technical and personnel limitations and aspiration was not performed on day 8 due to intense rain

\begin{tabular}{lccccc}
\hline & \multicolumn{2}{c}{ Adultrap } & & \multicolumn{2}{c}{ Aspirator } \\
\cline { 2 - 3 } \cline { 5 - 6 } Days & $\begin{array}{c}\text { Ae aegypti } \\
\text { male }\end{array}$ & $\begin{array}{c}\text { Ae. aegypti } \\
\text { female }\end{array}$ & & $\begin{array}{c}\text { Ae. aegypti } \\
\text { male }\end{array}$ & $\begin{array}{c}\text { Ae. aegypti } \\
\text { female }\end{array}$ \\
\hline 1 & 1 & 17 & & 34 & 21 \\
2 & 1 & 13 & & 39 & 28 \\
3 & 4 & 28 & & 32 & 23 \\
4 & 2 & 30 & & 32 & 17 \\
8 & 2 & 4 & & - & - \\
9 & 1 & 5 & & 49 & 18 \\
10 & 2 & 14 & & 33 & 7 \\
11 & 12 & 18 & & 124 & 73 \\
\hline Total & 25 & 129 & & 343 & 187 \\
\hline Mean per day & 3.12 & 16.12 & 49 & 26.71 \\
\hline
\end{tabular}

From the 129 Ae. aegypti females collected in the field by Adultrap, 125 (96.9\%) had their ovaries dissected in the laboratory. Of them, 117 (93.6\%) were gravid (Fig. 1A). Aspiration in houses where an Adultrap was installed collected 147 Ae. aegypti females, where $59.86 \%$ had ovaries in initial development stages (probably host-seeking females), $28.57 \%$ had ovaries in intermediary development stages and $11.56 \%$ were gravid. These data suggest that Adultrap had not collected all gravid females within a house (Fig. 1B). Aspiration in houses without Adultrap yielded 41 captured Ae. aegypti females, where $48.78 \%$ had ovaries in initial development stages, $29.26 \%$ had ovaries in intermediary stages and $21.25 \%$ were gravid (Fig. 1C).
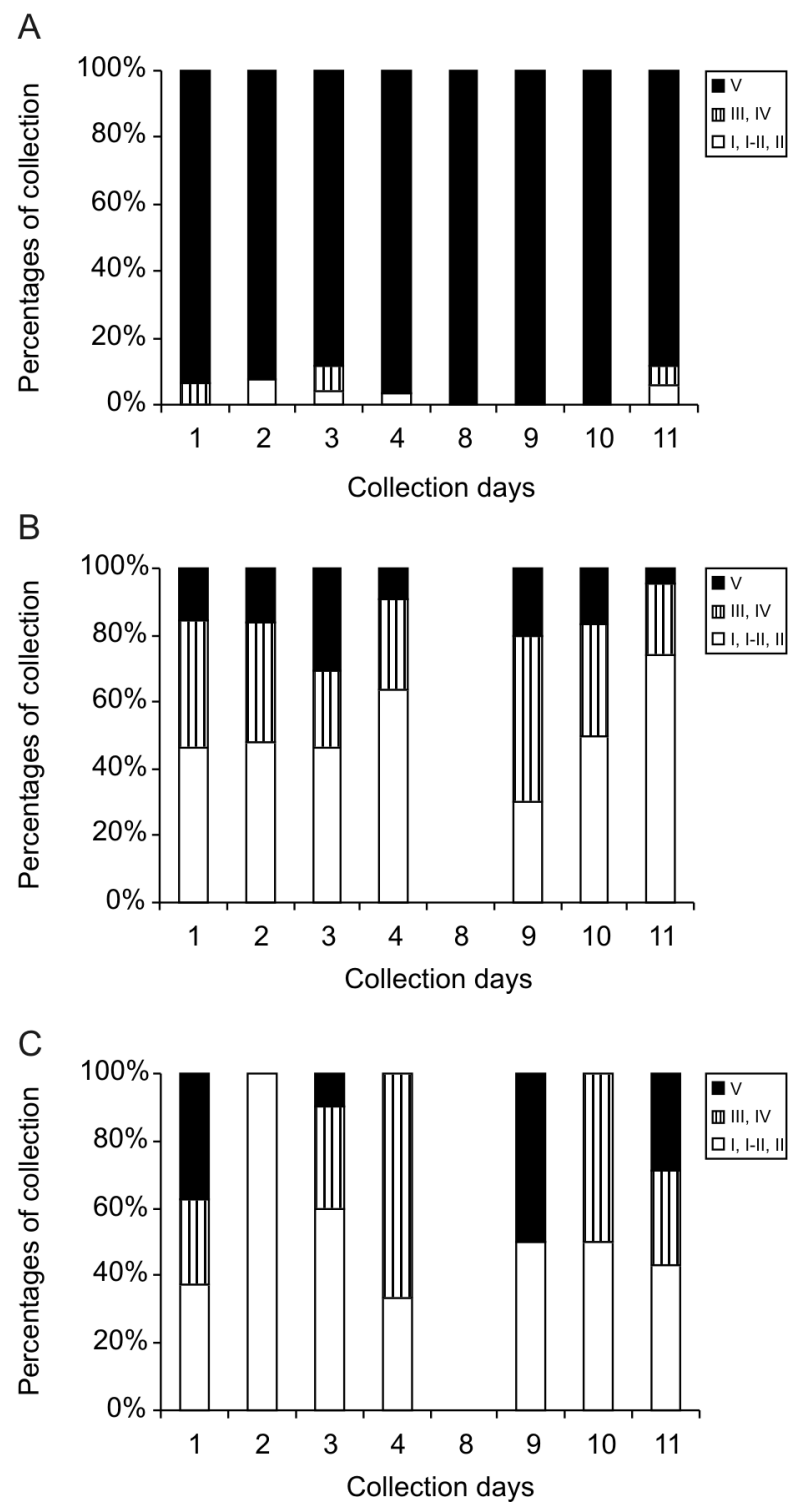

Fig. 1: variation of the ovarian development stage of Aedes aegypti females captured in Adultraps (A), aspirators in houses with Adultrap (B) and aspirators in houses without Adultrap (C) per day in the neighbor of Olaria, August 22nd - 1st September 2006. There were no collections on days 5, 6 and 7 due to technical and personnel limitations and aspiration was not performed on day 8 due to intense rain. 
The number of Ae. aegypti females collected by Adultrap did not seem to depend on whether the trap was placed in intra- or peridomestic areas, in sunlight or shadow, or whether it was baited with hay infusion or water alone. However, several other reports have shown that mosquito traps are more efficient when installed outdoors, since Ae. aegypti females generally tend to feed inside houses and rest and search for an oviposition site in the peridomestic area (Dibo et al. 2005, Maciel-deFreitas et al. 2006, Fávaro et al. 2006). In Rio de Janeiro, water-holding containers were generally more abundant outside houses (Maciel-de-Freitas et al. 2007). Therefore, it is possible that Adultraps installed indoors experienced less competition with nearby water containers, while outdoor Adultraps had a higher competition with the greater number of peridomestic containers. This relationship between Adultrap and water container abundance inside houses vs outdoors probably influenced the number of mosquitoes captured by traps in intra- and peridomestic areas.

In addition to its efficiency, Adultrap showed a high specificity for Ae. aegypti females, with $94 \%$ of captured individuals found to be gravid. However, the capture of additional gravid individuals by aspirators in houses where an Adultrap was installed (around $10-20 \%$ per day) suggests this trap did not capture all gravid females within a given house.

Starting on December 4th 2006, a mark, release and recapture (MRR) experiment was conducted in Olaria to compare Adultrap and MosquiTRAP efficiency under field conditions. MosquiTRAP was also designed to collect gravid Ae. aegypti females, but uses a synthetic attractant named AtrAedes. MosquiTRAP consists of a matte-black container with around $300 \mathrm{ml}$ of water and a black adhesive card in its interior to trap mosquitoes. The AtrAedes is also glued in the adhesive card. Ae. aegypti females used in MRR trials were derived from a laboratory colony that is renewed at least twice a year with field collected eggs in Rio de Janeiro. A total of 725 gravid and sugar-fed females, 6-7 days old, were marked with fluorescent dust and released outdoors at 08:0009:00 a.m. Recaptures started on the day following release; mosquitoes were collected three times a week for 18 days, using 96 Adultraps and 96 MosquiTRAPs (192 houses sampled per collection day) distributed over an area of $3.14 \mathrm{Km}^{2}$. Collected mosquitoes were identified and checked for the presence of the fluorescent mark with an UV light. The MRR experiment was approved by Fiocruz Ethical Committee - CEP/Fiocruz (protocol number 11591-2005).

A recapture rate of $6.34 \%(n=46)$ was observed with MosquiTRAP and Adultrap capturing 63.04 $(\mathrm{n}=29)$ and $36.96 \%(\mathrm{n}=17)$ of dust-marked mosquitoes, respectively. A total of 283 wild Ae. aegypti females were captured by MosquiTRAP and 200 by Adultrap. Thus, MosquiTRAP had higher capture efficiency than Adultrap in capturing wild Ae. aegypti females $\left(\chi^{2}=14.26\right.$; $\mathrm{df}=1 ; \mathrm{p}<0.05)$. From the 305 analyzed females collected in MosquiTRAPs, 205 (67\%) were trapped by the legs and 100 by the thorax (33\%).

When the efficiency of Adultrap was compared to MosquiTRAP, the latter presented significantly higher recapture rates, collecting a higher number of both wild and dust-marked Ae. aegypti females. However, despite the above differences, Adultrap and MosquiTRAP are both efficient and reliable traps for the capture of Ae. aegypti females in field conditions, and may perhaps become a new tool for dengue vector monitoring and control.

Finally, to evaluate the possible role of MosquiTRAP as an oviposition site a pupal survey was conducted during Adultrap and MosquiTRAP removal, after the end of the MRR experiment. From the 192 houses where a trap was installed, we searched Ae. aegypti immatures in only 157 premises. A total of 378 immature Ae. aegypti (40 pupae and 338 larvae) were collected in 43 positive houses, giving a HI of 27.38 and a Breteau Index of 29.93. The most productive containers were water tanks, MosquiTRAPs and plastic pots, with 42.5, 20.0 and $15 \%$ of the total pupae collected. Of the 96 MosquiTRAPs, 27 (28.12\%) were positive, containing 134 larvae and eight pupae after 18 days of exposure. Without considering the immature individuals collected in MosquiTRAP, the HI would have been 11.46 , i.e. a decrease of 2.38 times, from 27.38 to 11.46 . Interestingly, two MosquiTRAPs had immature Ae. aegypti in their interior but no adult females stuck to the glue card. Probably, the females had landed in a fraction of the card without sufficient glue and were able to lay their eggs without becoming trapped.

Overall, container productivity in Olaria was similar to that previously observed in slum and suburban neighborhoods of Rio de Janeiro (Maciel-de-Freitas et al. 2007), where containers used for water storage (e.g., water tanks) was highly productive. Remarkably, water tanks were highly productive in Olaria even with the regular piped water distribution in this neighborhood. Barrera et al. (1993) points to the role of residents' habits in maintaining infestation levels in some areas, as even people considered having an adequate supply of water kept numerous water storage containers. Thus, container productivity seems to be strongly related to community habits of storing water for routine use.

Adultrap seemed to be as efficient as backpack aspirators, but with higher sensitivity towards collecting $A e$. aegypti gravid females A higher mean number of $A e$. aegypti females was collected in MosquiTRAP than in Adultrap, but the former has the serious disadvantage of acting as a breeding site for dengue vectors. Overall, the potential of Adultrap and MosquiTRAP in monitoring the Ae. aegypti population is promising, but still needs to be evaluated through a longer time series and under different levels and patterns of infestation.

\section{ACKNOWLEDGEMENTS}

To Reginaldo Rego and Marcelo Celestino, for technical support and field collections, the Secretary of Health of Paraná, for providing the Adultraps, Ecovec, for providing the MosquiTRAPs, and the three anonymous referees for their comments.

\section{REFERENCES}

Barrera R, Ávila J, González-Tellez S 1993. Unreliable supply of potable water and elevated Aedes aegypti larval indices: a causal relationship? J Am Mosq Control Assoc 9: 189-195. 
Braga IA, Gomes AC, Nelson M, Mello RC, Bergamaschi DP, Souza JMP 2003. Comparação entre pesquisa larvária e armadilha de oviposição, para detecção de Aedes aegypti. Rev Soc Bras Med Trop 33: 347-353.

Christophers SR 1911. The development of the egg follicle in anophelines. Paludism 2: 73-89.

Dibo MR, Chiaravalloti-Neto F, Battigaglia A, Mondini A, Fávaro EA, Barbosa AAC, Glasser CM 2005. Identification of the best ovitrap installation sites for gravid Aedes (Stegomyia) aegypti in residences in Mirassol, state of São Paulo, Brazil. Mem Inst Oswaldo Cruz 100: 339-343.

Fávaro AE, Dibo MR, Mondini A, Ferreira AC, Barbosa AAC, Eiras AE, Barata EAMF, Chiaravalloti-Neto F 2006. Physiological state of Aedes (Stegomyia) aegypti mosquitoes captured with MosquiTRAPs in Mirassol, São Paulo, Brazil. J Vector Ecol 31: 285-291.

Focks DA. A review of entomological sampling method and indicators for dengue vectors. Special program for research and training in tropical diseases (TDR), UNICEF, UNDP, World Bank, World Health Organization. 2003 [cited 2008 Jul]. [40 pp.] Available from: http://www.who.int/tdr/publications/publications/pdf/ dengue_vectors.pdf.

Gama RA, Silva EM, Silva IM, Resende M, Eiras AE 2007. Evaluation of the sticky MosquiTRAP for detecting Aedes (Stegomyia) aegypti (L.) (Diptera: Culicidae) during the dry season in Belo Horizonte, Minas Gerais, Brazil. Neotrop Entomol 36: 294-302.

Gomes AC, Silva NN, Bernal RTI, Leandro AS, Camargo NJ, Silva AM, Ferreira AC, Ogura LC, Oliveira SJ, Moura SM 2007. Specificity of the Adultrap for capturing females of Aedes aegypti (Diptera: Culicidae). Rev Soc Bras Med Trop 40: 216-219.

Maciel-de-Freitas R, Eiras AE, Lourenço-de-Oliveira R 2006. Field evaluation of effectiveness of the BG-Sentinel, a new trap for capturing adult Aedes aegypti (Diptera: Culicidae). Mem Inst Oswaldo Cruz 101: 321-325.

Maciel-de-Freitas R, Marques WA, Peres RC, Cunha SP, Lourençode-Oliveira R 2007. Variation in Aedes aegypti (Diptera: Culicidae) container productivity in a slum and a suburban district of Rio de Janeiro during dry and wet seasons. Mem Inst Oswaldo Cruz 102: 489-496.

Silver JB 2008. Mosquito ecology - Field Sampling Methods. Springer, The Netherlands, $1498 \mathrm{pp}$.

SMS - Secretaria Municipal de Saúde 2008. www.saude.rio.rj.gov.br, accessed May 20, 2008.

Williams CR, Long SA, Russell RC, Ritchie SA 2006. Field efficacy of the BG-Sentinel compared with CDC Backpack Aspirators and $\mathrm{CO}_{2}$-baited EVS traps for collection of adult Aedes aegypti in Cairns, Queensland, Australia. J Am Mosq Control Assoc 22: 296-300. 